\title{
Total knee arthroplasty using a contemporary computer-assisted surgical system: a review of resection alignment on 7000 clinical cases
}

\author{
Yifei Dai $\mathrm{PhD}^{1 *}$, Cyril Hamad Dipl. Ing. ${ }^{2}$, Amaury Jung Dipl. Ing. ${ }^{2}$, and \\ Laurent Angibaud Dipl. Ing. ${ }^{1}$ \\ ${ }^{1}$ Exactech Inc, Gainesville, FL, 32653, USA \\ ${ }^{2}$ Blue-Ortho, Gieres, 38610, FR \\ yifei.dai@exac.com
}

\begin{abstract}
This study investigated the accuracy of Distal Femoral Cut and Proximal Tibial Cut during navigated total knee arthroplasty on a large cohort of patient. The findings demonstrated that CAOS is an accurate and precise solution to assist the surgeons achieve his/her surgical resection goals with substantial reduction of unacceptable resections.
\end{abstract}

\section{Introduction}

The long-term clinical success of the total knee arthroplasty (TKA) is critically dependent on the accurate positioning of the prosthesis. However, during TKAs performed using conventional mechanical instruments, surgical variability may undermine the surgeon's ability to achieve his/her surgical goals in terms of bony resections, the foundation of accurate component placement. Computerassisted orthopaedic surgery (CAOS) has been shown to offer increased accuracy and precision to the bony resections compared to the conventional techniques [1].

Previous studies of CAOS surgeries were mostly focused on the alignment outcomes based on limited number of patients from selected hospital sites [1]. In addition, several meta-analyses of the outcomes of CAOS systems have been reported [2,3]. However, these analyses did not differentiate between CAOS systems, while system-dependency has been reported in the alignment parameters [4]. In a previous study, we have benchmarked the ability of a specific CAOS system for its efficiency of achieving the intraoperatively defined surgical goals based a large number (4000+) of clinical cases [4].

The purpose of this study was to provide the updated assessment of the accuracy and precision of achieving surgical goals based a substantially expanded cohort of approximately 7000 cases using the contemporary CAOS system. 
Total Knee Arthroplasty Using a Contemporary Computer-Assisted Surgical System... Y. Dai et al.

\section{Materials and Methods}

Alignment parameters were extracted from the technical logs of 6888 TKA surgeries performed between October 2012 and January 2017 using a contemporary CAOS system (ExactechGPS, BlueOrtho, Grenoble, FR). The logs did not pertain any information related to the patients. The following surgical parameters were investigated: 1) planned resection, resection parameters defined by the surgeon prior to the bone cuts.

These parameters serve as inputs for the CAOS guidance; 2) Checked resection, digitalization of the realized resection surfaces by manually pressing an instrumented checker onto the bony cuts. Due to that anterior, posterior and chamfer cuts of the femur were all corresponded to the distal resection, only the distal resection was evaluated for the femur.

Deviations in the alignment between planned and checked resections were calculated in coronal and sagittal planes for both tibia and femur (planned vs checked). Acceptable resections were defined as resections with no more than $3^{\circ}$ of resection deviations.

\section{Results}

For the tibial resection, the deviations in coronal alignment (tibial varus/valgus angle) and sagittal alignment (posterior tibial slope) were $0.06 \pm 0.94^{\circ}$ and $-0.09 \pm 1.73^{\circ}$, respectively (Fig. $1 \mathrm{~A}$ ).

For the femoral resection, the deviations in coronal alignment (femoral varus/valgus angle) and sagittal alignment (femoral flexion) were $0.00 \pm 0.97^{\circ}$ and $-0.17 \pm 1.44^{\circ}$, respectively (Fig. $1 \mathrm{~B}$ ).

High percentages of the resections were found to be acceptable ( $>94 \%$ for alignment, Fig. 2).

\section{Discussion}

Numerous studies have shown that malalignment can lead to various complications, such as component loosening and instability, polyethylene wear, and patellar dislocation [8-10]. However, studies have reported that up to $20 \%$ of patients remain dissatisfied with the results of the surgery [11]. This may partially due to the fact that TKA performed using conventional instruments still largely relies on the surgeon's experience and skill level to ensure prosthesis position and orientation.

It has been shown that only $70-80 \%$ of the TKA cases can achieve satisfactory lower limb alignment (within $\pm 3^{\circ}$ of varus/valgus relative to the mechanical axis) [3,6].

In contrast, this study demonstrated that the use of the CAOS system assessed can provide an accurate and precise solution to assist the surgeons achieve his/her surgical resection goals with substantial reduction of unacceptable resections.

The strength of this study is that it summarized a large number of cases spanning the entire application history of the specific CAOS system, including both experienced users and new adopters of the technology. The data provided a complete clinical relevant evaluation demonstrating its high accuracy and precision in terms of bony resection alignment. 
Total Knee Arthroplasty Using a Contemporary Computer-Assisted Surgical System... Y. Dai et al.
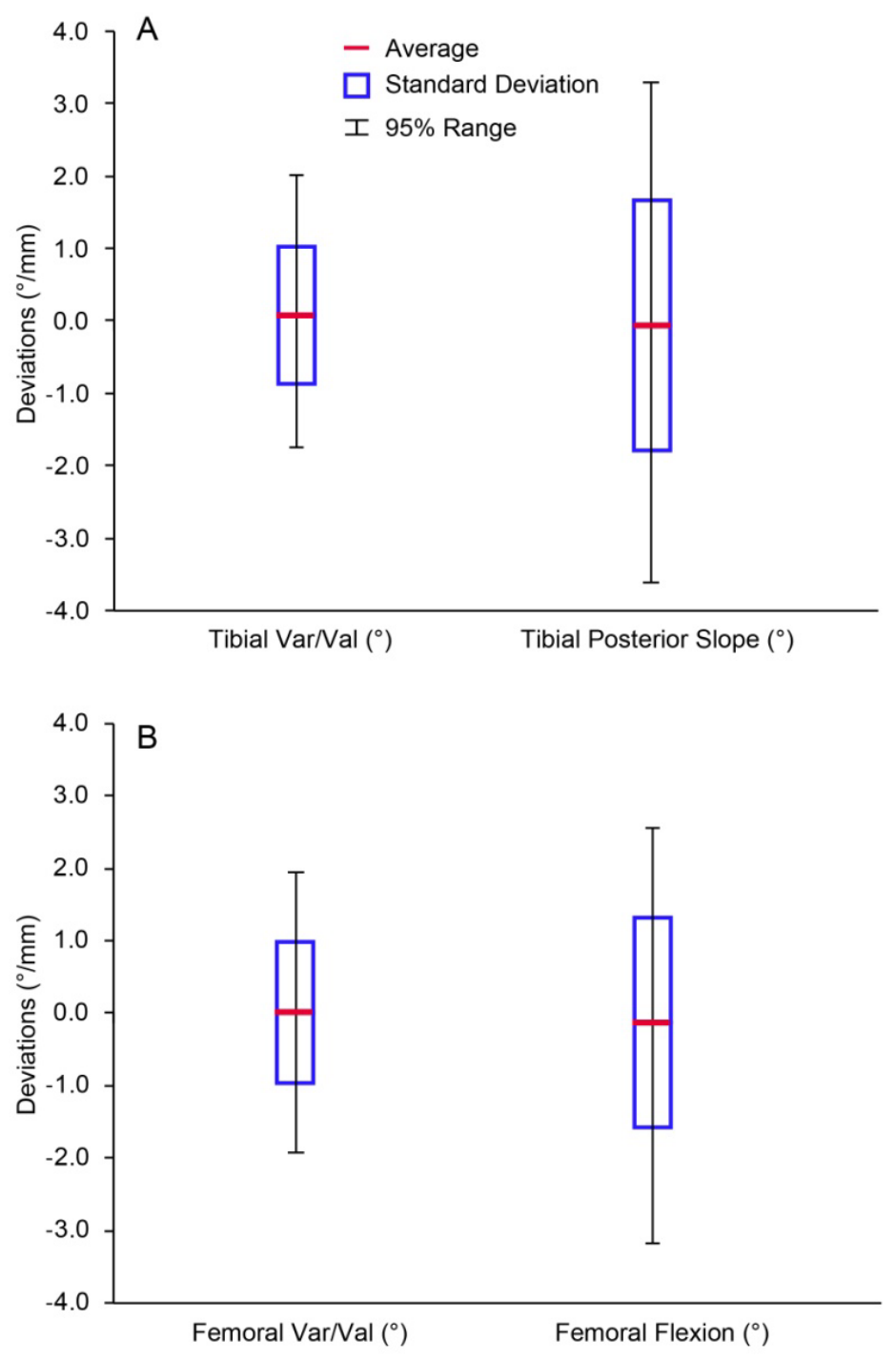

Positive value in deviation indicates that compared to the planned resection, the checked resection was more valgus and had less posterior slope (for tibia) or less flexion (for femur).

Figure 1 : A) tibial and B) femoral deviations (planned vs checked). Positive value in deviation indicates that compared to the planned resection, the checked resection was more valgus, and had less posterior slope (for tibia) or less flexion (for femur). 
Total Knee Arthroplasty Using a Contemporary Computer-Assisted Surgical System...

Y. Dai et al.
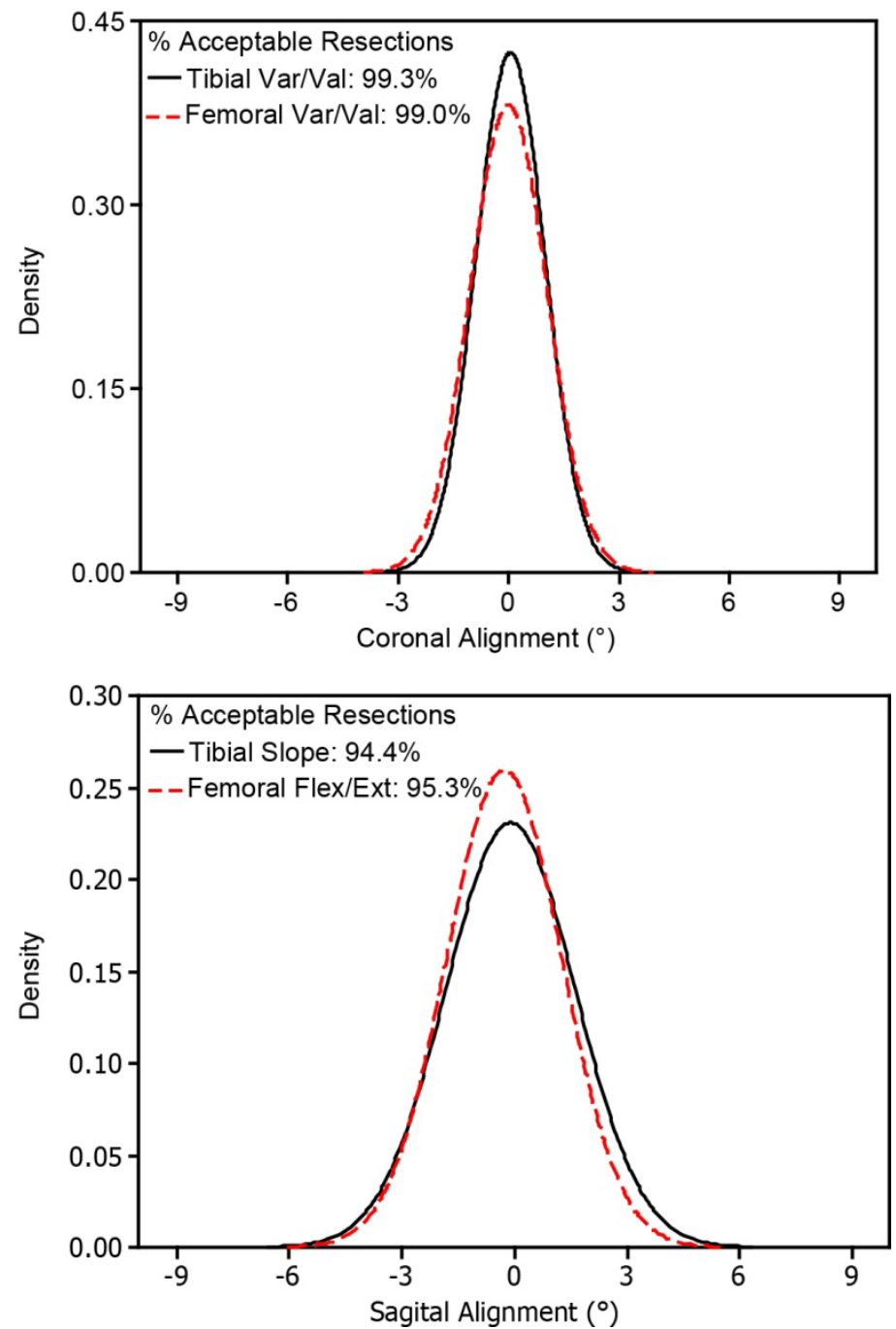

Figure 2 : Distribution of the resection deviations in A) coronal alignment and B) sagittal alignment, marked with the percentage of acceptable resections. 
Total Knee Arthroplasty Using a Contemporary Computer-Assisted Surgical System... Y. Dai et al.

\section{References}

[1] Sparmann M, et al. Positioning of total knee arthroplasty with and without navigation support: a prospective, randomised study. J Bone Joint Surg Br,85(6):830-5,2003.

[2]Brin YS, et al. Imageless computer assisted versus conventional total knee replacement. A Bayesian meta-analysis of 23 comparative studies. Int Orthop,35(3):331-9,2011.

[3] Hetaimish BM, et al. Meta-analysis of navigation vs conventional total knee arthroplasty. J Arthroplasty,27(6):1177-82,2012.

[4] Dai Y, et al. Total knee arthroplasty using a contemporary computer-assisted surgical system: a review of surgical parameters on more than 4000 clinical cases. CAOS 2016.

[5] Carli A, et al. Inconsistencies between navigation data and radiographs in total knee arthroplasty are system-dependent and affect coronal alignment. Can J Surg,57(5):305-13,2014.

[6] Ensini A, et al. Alignments and clinical results in conventional and navigated total knee arthroplasty. Clin Orthop Relat Res,457:156-62,2007.

[7] Bourne RB, et al. Patient satisfaction after total knee arthroplasty: who is satisfied and who is not? Clin Orthop Relat Res,468(1):57-63,2010.

[8] Jeffery RS, et al. Coronal alignment after total knee replacement. J Bone Joint Surg Br,73(5):709$14,1991$.

[9] Ritter A, et al. Postoperative alignment of total knee replacement: its effect on survival. Clin Orthop Relat Res,299:153-6,1994.

[10] Wasielewski RC, et al. Wear patterns on retrieved polyethylene tibial inserts and their relationship to technical considerations during total knee arthroplasty. Clin Orthop Relat Res, 299:31-43,1994. 